\title{
Tu N104 15
}

\section{Benefits of Blended Acquisition with Dispersed Source Arrays (DSA)}

\author{
M. Caporal* (Delft University of Technology) \& G. Blacquière (Delft \\ University of Technology)
}

\section{SUMMARY}

In blended data acquisition, the recorded wave field is incoherent. Nevertheless blended source units in the arrays are historically chosen to be equal. We propose to abandon this constraint. This allows us to suggest the exploitation of inhomogeneous blended sources, together representing a Dispersed Source Array (DSA). Each source unit involved in the survey might be dedicated to a narrow and arbitrary frequency bandwidth, without the need to satisfy the wideband requirement. Inhomogeneous blending with DSA has several attractive potential advantages. The design of such sources is potentially simpler. In addition, the DSA concept opens the possibility to use tow depths and spatial sampling intervals that are optimum for specific sources. The whole inhomogeneous ensemble of sources incorporated to the array is designed to cover the entire seismic bandwidth of interest. 


\section{Introduction}

Recent improvements in acquisition technology and research allow and encourage geophysicists and engineers to think outside the box. On this basis, since the last few decades, simultaneous and blended acquisition became more and more popular in seismic data survey design.

The benefits of using two or more shots overlapping during seismic surveys have been demonstrated (see, for example, Beasley et al., 1998; Beasley, 2008; Berkhout, 2008; Hampson et al., 2008; Berkhout and Blacquière, 2013). In simultaneous seismic data acquisition each subsurface grid point can be illuminated from a larger number of angles and, more importantly, from larger angles. This results in higher spatial resolution subsurface images and therefore potential better reservoir characterization. Survey time and costs are reduced as well and the total signal-to-noise ratio is also improved. Here with noise we mean ambient noise.

Furthermore, although blended source units in the arrays are historically chosen to be equal, the condition of employing only identical sources can be abandoned. This allows us to suggest the exploitation of inhomogeneous blended sources, together representing a Dispersed Source Array (DSA, refer to Berkhout, 2012). Each source unit involved in the survey might be dedicated to a narrow and arbitrary frequency bandwidth, without the need to satisfy the wideband requirement. The whole inhomogeneous ensemble of sources incorporated to the array is designed to cover the entire seismic bandwidth of interest. In the following paragraphs, the DSA concept is illustrated with some synthetic examples.

\section{Method and Theory}

To illustrate the DSA concept, four different source unit types are used: ultralow-, low-, mid- and highfrequency sources with band intervals 2-6, 5-15, 10-30 and 20-60 Hz, respectively. In such situation, given bandwidths are partially overlapping. However, the spectral properties of the sources are required to guarantee optimal amplitude conditions in the whole frequency band of interest. In Figure 1a the amplitude spectra of such sources are shown. Correspondent examples of acoustic energy propagation in a homogeneous medium are presented in Figure 1b.

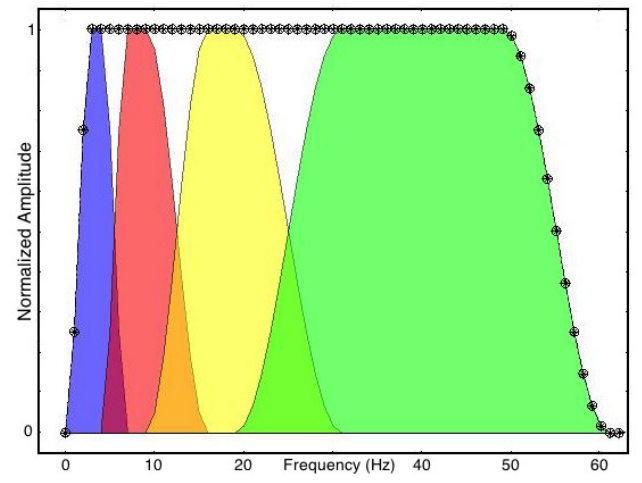

(a) Amplitude spectra correspondent to chosen ideal source units.
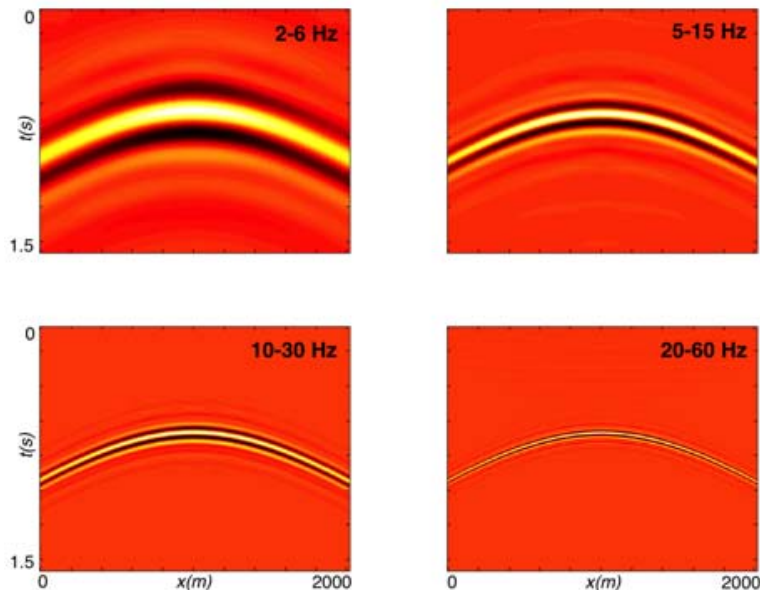

(b) Comparison of chosen narrowband source units response.

Figure 1: Four narrowband DSA source units.

Latest seismic vibrators and air-gun arrays are designed such that they transmit a wide bandwidth ranging over many octaves. From a practical point of view, a significant effort is required to profitably produce and operate such sources and we often need to accept a trade-off between wave transmission and system engineering efficiency. 


\section{Th. MADRID 2015}

We believe that blended DSA acquisition has the potential to relax the low versus high frequency compromise. Narrowband source units can be technically simpler to produce and more effective from an acoustic energy transmission point of view than today's broadband alternatives. The signal emission properties of each source unit can, in fact, be separately optimized for the specific frequency range. Operational flexibility may also improve. Modern multiple-driver loudspeaker systems are based on the same key concept and their improved performances are demonstrated (Borwick, 2001). The overlap in the bandwidths among different sources is relatively small, potentially simplifying the treatment of the data both for deblending and eventually direct processing.

Moreover, according to the Nyquist-Shannon sampling theorem, the ideal source spacing should not be larger than a half-period of the smallest horizontal wave length (Niland, 1989; Vermeer, 2012). The sampling rule for spatial sampling is

$$
\Delta x \leq \frac{v_{\min }}{2 f_{\max } \sin \theta_{\max }}
$$

Where $v_{\min }$ is the minimum propagation velocity while $f_{\max }$ and $\theta_{\max }$ are the maximum frequency and the maximum angle we aim to record, respectively. Therefore, the sampling density requirements depend on the maximum emitted frequency. In the case of different bandwidth sources, this means that each type of source has its own optimal maximum spacing, which is larger for the low-frequencies sources and smaller for the high-frequencies sources. Hence, the required number of low-frequencies source units (shots) is lower than the required number of high-frequencies source units (shots). In Table 1, the minimum number of regularly spaced source points needed to adequately cover the same 2D line (approximately $1200 \mathrm{~m}$ long) is reported for each source unit type.

\begin{tabular}{|c|c|c|}
\hline Frequency band & Number of source points & $\begin{array}{c}\text { Optimal (Nyquist) sampling interval } \\
\text { (For acoustic energy propagation in water, } \\
\left.\theta_{\max }=90^{\circ}\right)\end{array}$ \\
\hline $\begin{array}{c}2-6 \mathrm{~Hz} \\
\text { ultralow }\end{array}$ & 10 & $125 \mathrm{~m}$ \\
\hline $\begin{array}{c}5-15 \mathrm{~Hz} \\
\text { low }\end{array}$ & 25 & $50 \mathrm{~m}$ \\
\hline $\begin{array}{c}10-30 \mathrm{~Hz} \\
\text { mid }\end{array}$ & 50 & $25 \mathrm{~m}$ \\
\hline $\begin{array}{c}20-60 \mathrm{~Hz} \\
\text { high }\end{array}$ & 100 & $12.5 \mathrm{~m}$ \\
\hline
\end{tabular}

Table 1: Number of source points and sampling interval for the different source units. Survey line is approximately $1200 \mathrm{~m}$ long.

Extra benefits arise if we look at the source ghost issue. For an exhaustive explanation of the problem, the reader is referred to Parkes and Hatton (1986). To reduce the effect of the ghost, each source type can be placed at the optimum depth below the water surface, e. g. at $d=\lambda / 4$, one quarter of its central frequency wavelength $\lambda$. We could see this proposal as an extension of the multi-level source arrays concept already widely developed and tested in the oil industry (see, for an overview, Shen et al., 2014). In this way, ghost destructive interference and notches are largely avoided. Low frequency attenuation due to a too shallow tow depth is also avoided. Nevertheless, in the higher angles, ghost interference still causes loss of information that can be fixed with deghosting algorithms potentially simpler than today's ones. All these effects are shown in Figure 2 and in Figure 3. 


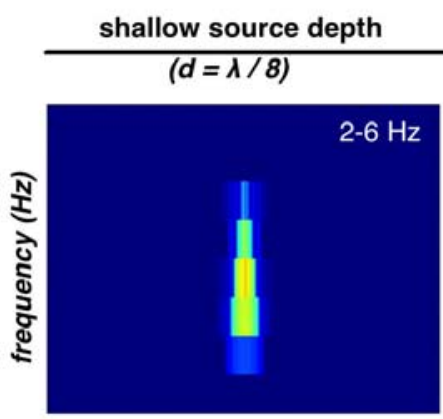

optimal source depth
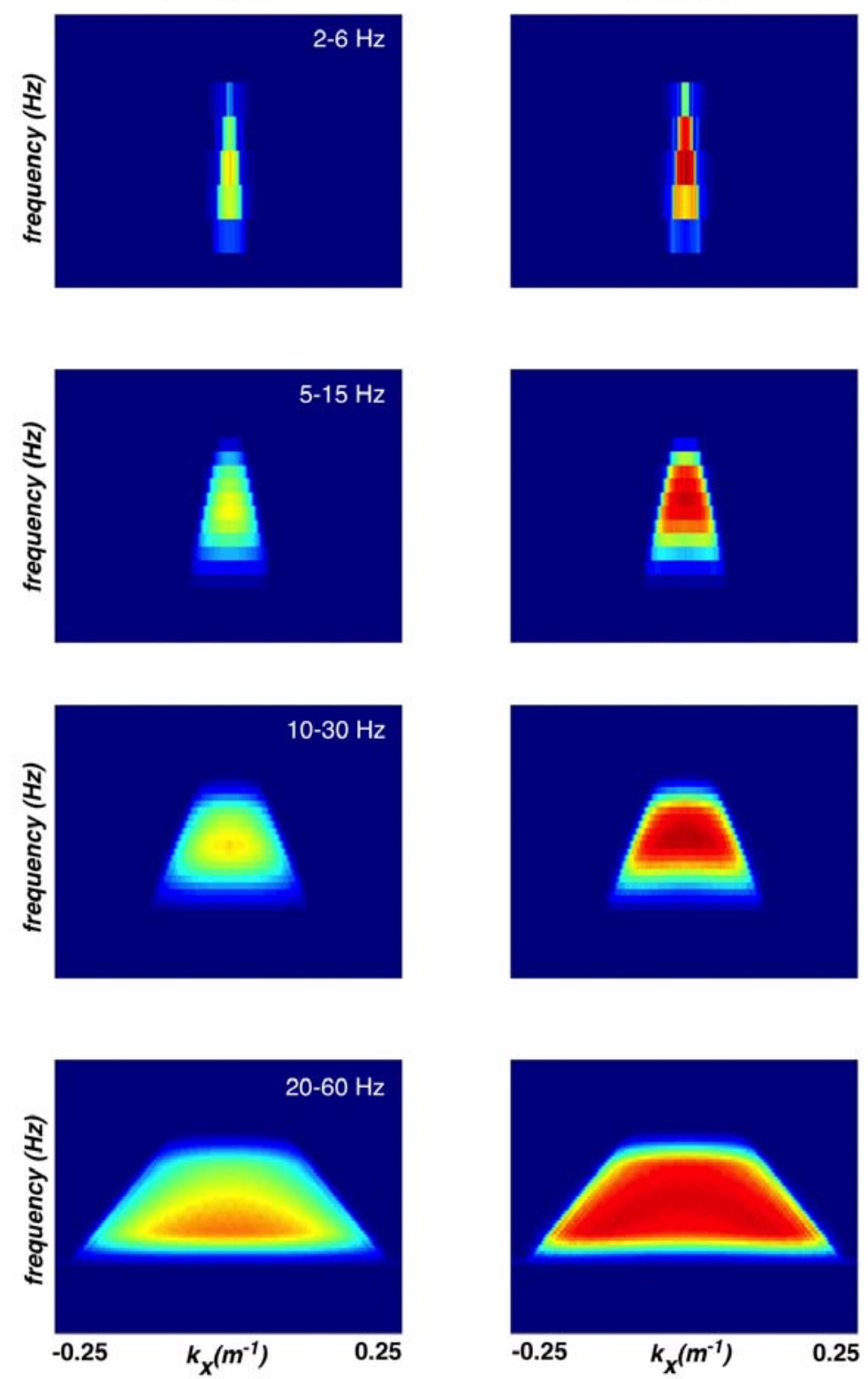

(1) MADRID 2015

1-4 June 2015 I IFEMA Madrid

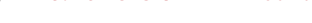

deep source depth
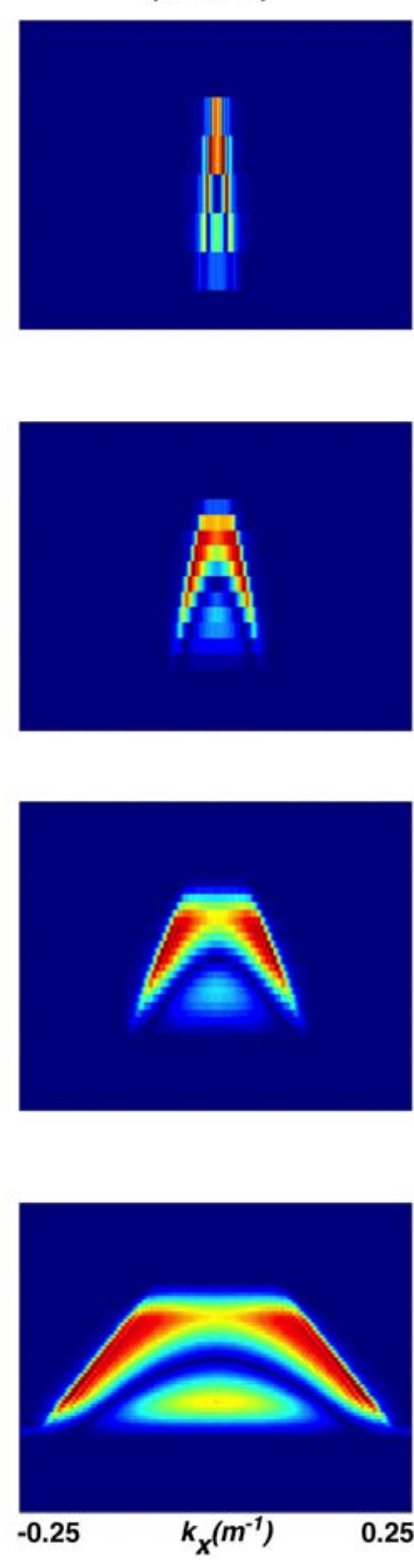

Figure 2: In case of shallow source depths, lower frequencies are attenuated (left column). When sources are towed deep, ghost spectral notches appear (right column). DSA concept reduces both issues (middle column).
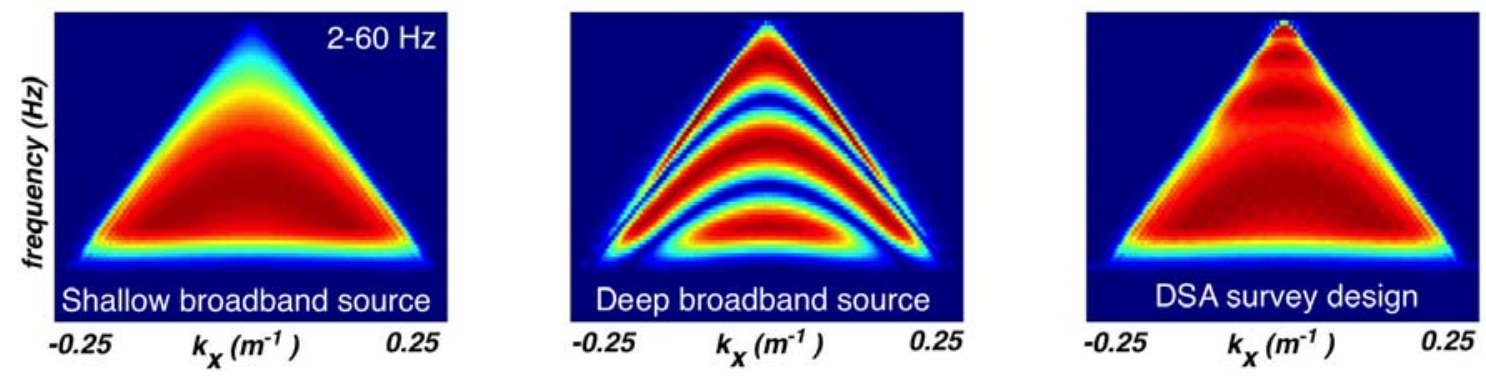

Figure 3: Results for the full bandwidth are shown. 


\section{Conclusions}

By combining the blending principle and the DSA concept, it is possible to make some fundamental improvements by changing the system architecture. The design of narrowband sources, which together cover the total seismic bandwidth, may become significantly simpler. Additionally, the source density could be chosen in a frequency dependent manner. Applying this concept, surveys may be carried out by acquisition systems that are less complex and more flexible than the ones that are used today. Furthermore, the possibility to use tow depths that are optimum for specific sources gives extra benefits if we look at the source ghost issue. Ghost destructive interference and low frequency attenuation due to shallow tow depths are largely avoided. Considering the aforementioned several potential advantages, we plan to carry this research forward quantitatively in the near future.

\section{Acknowledgements}

The authors would like to thank the sponsors of the Delphi consortium for the stimulating discussions during the Delphi meetings and the continuing financial support.

\section{References}

Beasley, C.J. [2008] A new look at marine simultaneous sources. The Leading Edge, 27(7), 914-923.

Beasley, C.J., Chambers, R.E. and Jiang, Z. [1998] A new look at simultaneous sources. SEG, Soc. Expl. Geophys., Expanded abstracts, New Orleans, 133-135.

Berkhout, A.J. [2008] Changing the mindset in seismic acquisition. The Leading Edge, 27(7), 924-938.

Berkhout, A.J. [2012] Blended acquisition with dispersed source arrays. Geophysics, 77(4), A19-A23.

Berkhout, A.J. and Blacquière, G. [2013] Effect of noise in blending and deblending. Geophysics, 78(5), A35A38.

Borwick, J. [2001] Loudspeaker and Headphone Handbook. Focal Press.

Hampson, G., Stefani, J. and Herkenhoff, E.F. [2008] Acquisition using simultaneous sources. The Leading Edge, 27(7), 918-923.

Niland, R.A. [1989] Optimum oversampling. J. Acoust. Soc. Amer., 86(5), 1805-1812.

Parkes, G. and Hatton, L. [1986] The Marine Seismic Source. Reidel.

Shen, H., Elboth, T., Tian, G., Warszawski, J. and Lilja, D. [2014] Theoretical study on multi-level source design. Geophysical Prospecting, 62(6), 1337-1352.

Vermeer, G.J.O. [2012] 3D Seismic Survey Design. SEG Books. 